\title{
Robust Adaptive Regulation of Polynomial Systems with Dynamic Uncertainties
}

\author{
Zhiyong Chen and Jie Huang
}

\begin{abstract}
This paper studies the global regulation problem for a class of nonlinear polynomial systems subject to both dynamic uncertainty and static uncertainty. The dynamic uncertainty does not vanish at the origin of the state space and thus is not input-to-state stable (ISS). As a result, the small gain theory based robust control technique alone cannot handle this problem. We manage to integrate both robust control and adaptive control techniques to overcome the difficulty. The problem studied here is motivated from the global robust servomechanism problem for the lower triangular systems subject to an uncertain exosystem. An example is given to illustrate this point.
\end{abstract}

Index Terms-Robust control, Adaptive control, Nonlinear systems, Regulation, Stabilization

\section{INTRODUCTION}

Consider a general nonlinear system of the cascaded form as follows:

$$
\begin{aligned}
\dot{x}_{o}= & f_{o}\left(x_{o}, x_{1}, d\right) \\
\dot{z}_{i}= & M_{i} z_{i}+\gamma_{i}\left(\zeta_{i-1}, \chi_{i}, d\right)+g_{i}\left(\zeta_{i-1}, \chi_{i}, d\right) \\
\dot{x}_{i}= & f_{i}\left(\zeta_{i}, \chi_{i}, d\right)+p_{i}\left(\zeta_{i}, \chi_{i}, d\right)+x_{i+1}, \\
& i=1, \cdots, r \\
\mathcal{Y}= & \mathcal{M}\left(\chi_{r}, \zeta_{r}, d\right) \\
e= & x_{1}
\end{aligned}
$$

where $x_{o} \in \mathbb{R}^{n_{o}}, x_{i} \in \mathbb{R}, z_{i} \in \mathbb{R}^{n_{i}}, \chi_{i}:=\operatorname{col}\left(x_{o}, \cdots, x_{i}\right)$, $\zeta_{i}:=\operatorname{col}\left(z_{1}, \cdots, z_{i}\right), f_{i}(0,0, d)=0$, and $\gamma_{i}(0,0, d)=0$. In the system $(1.1),\left(\chi_{r}, \zeta_{r}\right)$ is the state vector, $u:=x_{r+1}$ the input, $\mathcal{Y} \in \mathbb{R}^{m}$ the measurable output that can be used by feedback control law, and $e$ the output to be regulated. The function $d(t)$ is bounded and sufficiently smooth in $t$ representing unknown parameters and/or disturbances, called static uncertainty. On the other hand, the dynamics governing $\zeta_{r}$ are called dynamic uncertainty in the sense that the state $\zeta_{r}$ cannot be used for feedback control. Since we assume the state $\zeta_{r}$ is not available for feedback and the state $\chi_{r}$ is available for feedback control, the measurement output $\mathcal{Y}$ contains $\chi_{r}$ as its component and contains no components of $\zeta_{r}$. Also, we assume $M_{i}$ is a Hurwitz matrix, and all functions are polynomial in their variables. To distinguish

The work of the first author was supported by the Australian Research Council under grant No. DP0878724. The work of the second author was supported by the Research Grants Council of the Hong Kong Special Administration Region under grant No. 412007.

Z. Chen is with the School of Electrical Engineering and Computer Science, The University of Newcastle, Callaghan, NSW 2308, Australia zhiyong. chen@newcastle.edu.au

J. Huang is with the Department of Mechanical and Automation Engineering, The Chinese University of Hong Kong, Shatin, N.T., Hong Kong jhuang@mae.cuhk.edu.hk the different role played by the functions $\gamma_{i}$ and $g_{i}$, and the functions $f_{i}$ and $p_{i}$, we assume $g_{i}\left(\zeta_{i-1}, \chi_{i}, d\right)$ and $p_{i}\left(\zeta_{i-1}, \chi_{i}, d\right)$ are not identically zero.

The class of feedback control laws adopted in this paper is of the following form

$$
u=\varkappa_{1}\left(x_{c}, \mathcal{Y}\right), \quad \dot{x}_{c}=\varkappa_{2}\left(x_{c}, \mathcal{Y}\right)
$$

where $x_{c} \in \mathbb{R}^{n_{c}}$ with $n_{c}$ a nonnegative integer to be specified later, and the functions $\varkappa_{1,2}$ are polynomial. Now, it is ready to describe the problem as follows.

Global Regulation Problem. Design a control law of the form (1.2) such that the trajectories of the closed-loop system composed of (1.1) and (1.2) are bounded for all initial conditions, and the performance output $e$ converges to zero asymptotically.

A special case of the above problem where

$$
g_{i}\left(\zeta_{i-1}, \chi_{i}, d\right)=0, p_{i}\left(\zeta_{i}, \chi_{i}, d\right)=0,
$$

has been studied in several papers [1], [2], [3], [4]. For this special case, the problem can be dealt with using robust control approach. In particular, we note that, for this special case, the system (1.1) arises naturally from studying the global robust servomechanism problem (or called an output regulation problem alternatively) of a class of lower triangular systems with a known exosystem where the static uncertainty $d(t)$ modeling both the time-varying exogenous signals and the constant unknown parameter, and the dynamic uncertainty models the internal model [3], [5], [6], [7], [8]. In this paper, we will consider the more general case with $p_{i}(0,0, d) \neq 0$ and $g_{i}(0,0, d) \neq 0$ for some $d(t)$. The specific form of system (1.1) is extracted from the global robust servomechanism problem of lower triangular nonlinear systems subject to an uncertain linearly neutrally stable exosystem. When the exosystem contains uncertain parameter, the terms $g_{i}$ and $p_{i}$ will be present to reflect the mismatch between the actual unknown parameter and its estimated value in the exosystem.

For convenience, in what follows, we refer to the terms $p_{i}$ and $g_{i}$ as equilibrium point perturbing (e.p.p.) terms for the reason that these terms account for the perturbations of the equilibrium point due to the disturbance $d$. An implication of the presence of e.p.p. terms is that the dynamic uncertainty is now not ISS any more. This fact complicates the solution of the above problem. To make the problem more tractable, we need to make some assumptions on the e.p.p. terms and the measurement output. Let $d(t):=\operatorname{col}(v(t), \mu)$ where $v(t) \in$ $\mathbb{R}^{q}$ represents the time varying disturbances and $\mu \in \mathbb{R}^{p}$ the unknown constant parameters. Then, we assume: 
Assumption 1.1: For $i=1,2, \cdots, r$, there exist polynomial functions $m_{i}, \bar{m}_{i}, h_{i}, l_{i}$ such that,

$$
g_{i}\left(\zeta_{i-1}, \chi_{i}, d\right)=h_{i}\left(\bar{y}_{i}, \mu\right), \quad p_{i}\left(\zeta_{i}, \chi_{i}, d\right)=l_{i}\left(y_{i}, \mu\right)
$$

where $y_{i}=m_{i}\left(\zeta_{i}, \chi_{i}, d\right)$ and $\bar{y}_{i}=\bar{m}_{i}\left(\zeta_{i-1}, \chi_{i}, d\right)$.

Assumption 1.2: For $i=1,2, \cdots, r, y_{i}$ and $\bar{y}_{i}$ are measurable, i.e., they are components of $\mathcal{y}$. Moreover, for $i=1,2, \cdots, r-1, \dot{y}_{i}=\varkappa_{i}\left(y_{i+1}, \mu\right)$ for a polynomial function $\varkappa_{i}$.

A special case of system (1.1) is considered in [2] where a robust adaptive approach is developed to deal with a special type of e.p.p. terms. More precisely, the system studied in [2] satisfies the following conditions: (a) The overall dynamic uncertainty has the ISS property with input $x_{1}$ (not $\chi_{r}$ ) which essentially assumes the dynamic uncertainty only appears on the top of the plant. (b) The dynamic uncertainty does not contain e.p.p. terms, i.e., $g_{i}=0$, for $i=1, \cdots, r$. (c) When the exact adaptive regulation is studied, only the case $r=2$ is studied, and $p_{2}\left(\zeta_{2}, \chi_{2}, d\right)$ is bounded by a function independent of $x_{2}$. These three technical assumptions are vital for the robust adaptive controller design proposed in [2]. The generality of our problem poses some specific challenges as follows.

(i) As $z_{i}$ is not available for feedback, the standard adaptive control technique as used in [2] cannot handle the e.p.p. term $g_{i}$ appearing in the subsystem governing $z_{i}$. We have devised a dynamic coordinate transformation technique to overcome this difficulty.

(ii) When $r>1$, the dynamic transformation technique mentioned in the last paragraph has to be applied recursively. Each application of the dynamic transformation will lead to an extended system in a form similar to system (1.1) but with much more complex e.p.p. terms. This phenomenon will be called "propagation of uncertainty". Fortunately, for polynomial systems, we will show that at each recursive step, the e.p.p. terms may satisfy the linearly parameterization condition like Assumption 1.1 providing that a certain measurement output can be defined. As a result, an adaptive law can be constructed. It should be noted that the problem in [2] does not encounter this complication due to the aforementioned assumption (c).

\section{PReliminaries}

As the system (1.1) involves both static and dynamic uncertainties and the dynamic uncertainty does not satisfy the ISS assumption. These complexities entail an approach that integrates both robust and adaptive techniques. As always, the key for developing an adaptive control law is to find an appropriate Lyapunov function candidate for the system to be controlled. This observation necessitates the study of the special case of (1.1) where (1.3) is satisfied. For this special case, an explicitly constructed Lyapunov function can be found in [4], [9]. In this section, we will summarize the results of paper [4]. For this purpose, we list an assumption as follows:
Assumption 2.1: There exists a polynomial function $U_{o}\left(x_{o}\right)$ bounded by some class $\mathcal{K}_{\infty}$ polynomial functions of $\left\|x_{o}\right\|$, such that, along the trajectories of $\dot{x}_{o}=f_{o}\left(x_{o}, x_{1}, d\right)$,

$$
\frac{d U_{o}\left(x_{o}\right)}{d t} \leq-\left\|x_{o}\right\|^{2}+\pi\left(x_{1}\right)
$$

for some polynomial positive definite function $\pi$.

Let $\alpha_{i}\left(x_{i}\right), i=1, \cdots, r$, be some sufficiently smooth functions. Applying the following coordinate transformation

$$
\begin{array}{r}
\tilde{x}_{o}=x_{o}, \tilde{x}_{1}=x_{1}, \tilde{x}_{i+1}=x_{i+1}-\alpha_{i}\left(\tilde{x}_{i}\right), \\
i=1, \cdots, r
\end{array}
$$

to the system (1.1)+(1.3) gives

$$
\begin{aligned}
\dot{x}_{0} & =f_{0}\left(x_{o}, \tilde{x}_{1}, d\right) \\
\dot{z}_{i} & =M_{i} z_{i}+\varphi_{i}\left(\zeta_{i-1}, \tilde{\chi}_{i}, d\right) \\
\dot{\tilde{x}}_{i} & =\phi_{i}\left(\zeta_{i}, \tilde{\chi}_{i}, d\right)+\alpha_{i}\left(\tilde{x}_{i}\right)+\tilde{x}_{i+1}, i=1, \cdots, r
\end{aligned}
$$

where $\tilde{\chi}_{i}=\operatorname{col}\left(x_{o}, \tilde{x}_{1}, \cdots, \tilde{x}_{i}\right)$ and

$$
\begin{aligned}
\varphi_{i}\left(\zeta_{i-1}, \tilde{\chi}_{i}, d\right)= & \gamma_{i}\left(\zeta_{i-1}, x_{o}, \tilde{x}_{1}, \tilde{x}_{2}+\alpha_{1}\left(\tilde{x}_{1}\right)\right. \\
& \left.\tilde{x}_{i}+\alpha_{i-1}\left(\tilde{x}_{i-1}\right), d\right) \\
\phi_{i}\left(\zeta_{i}, \tilde{\chi}_{i}, d\right)= & f_{i}\left(\zeta_{i}, x_{o}, \tilde{x}_{1}, \tilde{x}_{2}+\alpha_{1}\left(\tilde{x}_{1}\right), \cdots\right. \\
& \left.\tilde{x}_{i}+\alpha_{i-1}\left(\tilde{x}_{i-1}\right), d\right) \\
& -\left(\partial \alpha_{i-1}\left(\tilde{x}_{i-1}\right) / \partial \tilde{x}_{i-1}\right) \times \\
& \left(\phi_{i-1}\left(\tilde{\zeta}_{i-1}, \tilde{\chi}_{i-1}, d\right)+\right. \\
& \left.\alpha_{i-1}\left(\tilde{x}_{i-1}\right)+\tilde{x}_{i}\right) .
\end{aligned}
$$

In particular, $\phi_{1}\left(\zeta_{1}, \tilde{\chi}_{1}, d\right)=f_{1}\left(\zeta_{1}, \chi_{1}, d\right)$.

The following theorem is from [4] with a slight modification for the need of this paper. A direct implication of this theorem is that the static controller $u=\alpha_{r}\left(\tilde{x}_{r}\right)$ globally stabilizes the system $(1.1)+(1.3)$ with $V\left(\zeta_{r}\right)+W\left(\tilde{\chi}_{r}\right)$ as an Lyapunov function for the closed-loop system.

Theorem 2.1: Under Assumption 2.1, there exist polynomial functions $\alpha_{i}(\cdot), i=1, \cdots, r$, and positive definite and radially unbounded functions $V\left(\zeta_{r}\right)$ and $W\left(\tilde{\chi}_{r}\right)=$ $\sum_{i=0}^{r} W_{i}\left(\tilde{x}_{i}\right)$, such that, along the trajectories of the system $(2.3)+(1.3)$ with $\tilde{x}_{r+1}=0$,

$$
\frac{d\left(V\left(\zeta_{r}\right)+W\left(\tilde{\chi}_{r}\right)\right)}{d t} \leq-k\left(\zeta_{r}, \tilde{\chi}_{r}\right)
$$

for some positive definite function $k(\cdot, \cdot)$.

\section{MAIN RESUlT}

The major difficulty in solving the problem is to deal with the e.p.p. terms appearing in the dynamic uncertainty. Our idea is to develop a dynamic coordinate transformation technique to obtain an extended system whose dynamic uncertainty does not contain the e.p.p. terms and is thus input-to-state stable. As a result, the global regulation problem of the resulting extended system can be handled by an adaptive control technique. To introduce the adaptive technique, we require some uncertain functions to be linearly parameterized. For this purpose, in addition to Assumption 1.1 , we need two more assumptions. These two assumptions 
can be satisfied in many situations and an example is given in Section IV.

Assumption 3.1: There exists a polynomial function $\bar{f}_{i}$ such that

$$
\bar{f}_{i}\left(y_{i}, \zeta_{i}-\bar{\zeta}_{i}, \chi_{i}, \bar{\chi}_{i}, \mu\right)=f_{i}\left(\zeta_{i}, \chi_{i}, d\right)-f_{i}\left(\bar{\zeta}_{i}, \bar{\chi}_{i}, d\right),
$$

for all $\zeta_{i}, \bar{\zeta}_{i}, \chi_{i}, \bar{\chi}_{i}$.

Assumption 3.2: There exists a polynomial function $\bar{\gamma}_{i}$ such that

$\bar{\gamma}_{i}\left(\bar{y}_{i}, \zeta_{i-1}-\bar{\zeta}_{i-1}, \chi_{i}, \bar{\chi}_{i}, \mu\right)=\gamma_{i}\left(\zeta_{i-1}, \chi_{i}, d\right)-\gamma_{i}\left(\bar{\zeta}_{i-1}, \bar{\chi}_{i}, d\right)$,

for all $\zeta_{i-1}, \bar{\zeta}_{i-1}, \chi_{i}, \bar{\chi}_{i}$.

As the dynamic coordinate transformation technique to be introduced will incur some additional notations, we will first study the case where $r=1$ to better reveal our idea. This special case will also become the first step of the general case which is handled by a recursive method.

\section{A. The Case with $r=1$}

When $r=1$, the system (1.1) is reduced to the following form:

$$
\begin{aligned}
& \dot{x}_{o}=f_{o}\left(x_{o}, x_{1}, d\right) \\
& \dot{z}_{1}=M_{1} z_{1}+\gamma_{1}\left(\chi_{1}, d\right)+h_{1}\left(\bar{y}_{1}, \mu\right) \\
& \dot{x}_{1}=f_{1}\left(z_{1}, \chi_{1}, d\right)+l_{1}\left(y_{1}, \mu\right)+u .
\end{aligned}
$$

As the state $z_{1}$ is not measurable for feedback, there is no clue how to handle the term $h_{1}\left(\bar{y}_{1}, \mu\right)$ using the adaptive control technique. To overcome this difficulty, we note that, by Assumption 1.1, $h_{1}$ is a polynomial function. Thus we can let

$$
h_{1}\left(\bar{y}_{1}, \mu\right)=\varrho_{1}\left(\bar{y}_{1}\right) \varpi_{1}(\mu)
$$

for a sufficiently smooth function matrix $\varrho_{1}$ and a column function vector $\varpi_{1}$.

Now, we will move the unknown parameters represented by $\varpi_{1}(\mu)$ in (3.2) to the subsystem (3.3). To this end, define a dynamic coordinate transformation as follows. Let $s_{1}$ be a state matrix with the same dimension as that of $\varrho_{1}$, and let

$$
\dot{s}_{1}=M_{1} s_{1}+\varrho_{1}\left(\bar{y}_{1}\right) .
$$

Then, the coordinate transformation $\bar{z}_{1}=z_{1}-s_{1} \varpi_{1}(\mu)$ gives

$$
\begin{aligned}
\dot{\bar{z}}_{1}= & M_{1} z_{1}+\gamma_{1}\left(\chi_{1}, d\right)+\varrho_{1}\left(\bar{y}_{1}\right) \varpi_{1}(\mu) \\
& -\left[M_{1} s_{1}+\varrho_{1}\left(\bar{y}_{1}\right)\right] \varpi_{1}(\mu) \\
= & M_{1} \bar{z}_{1}+\gamma_{1}\left(\chi_{1}, d\right) .
\end{aligned}
$$

We note the term $h_{1}\left(\bar{y}_{1}, \mu\right)$ disappears from the subsystem (3.6).

Under this dynamic coordinate transformation, we have

$$
\dot{x}_{1}=f_{1}\left(\bar{z}_{1}, \chi_{1}, d\right)+\ell_{1}\left(y_{1}, s_{1}, \mu\right)+u
$$

where

$$
\ell_{1}\left(y_{1}, s_{1}, \mu\right)=f_{1}\left(z_{1}, \chi_{1}, d\right)-f_{1}\left(\bar{z}_{1}, \chi_{1}, d\right)+l_{1}\left(y_{1}, \mu\right)
$$

due to Assumption 3.1 with $i=1$. Because the function $\ell_{1}\left(y_{1}, s_{1}, \mu\right)$ is polynomial, it is linearly parameterized in the sense that

$$
\ell_{1}\left(y_{1}, s_{1}, \mu\right)=\rho_{1}\left(y_{1}, s_{1}\right) \omega_{1}(\mu)
$$

for a sufficiently smooth row vector function $\rho_{1}$ and a column function vector $\omega_{1}$.

Now, the $x_{1}$-subsystem is in the following form:

$$
\dot{x}_{1}=f_{1}\left(\bar{\zeta}_{1}, \chi_{1}, d\right)+\rho_{1}\left(y_{1}, s_{1}\right) \omega_{1}(\mu)+u .
$$

And it is ready to choose the following controller,

$$
u=-\rho_{1}\left(y_{1}, s_{1}\right) \hat{\omega}_{1}+\alpha_{1}\left(x_{1}\right),
$$

where the function $\alpha_{1}(\cdot)$ is the one in Theorem 2.1, and the vector $\hat{\omega}_{1}$ is used to estimate $\omega_{1}(\mu)$. In particular, the vector $\hat{\omega}_{1}$ is generated by an update law

$$
\dot{\hat{\omega}}_{1}=\psi_{1}\left(y_{1}, s_{1}\right)=k_{1}\left(d W_{1}\left(x_{1}\right) / d x_{1}\right) \rho_{1}^{\top}\left(y_{1}, s_{1}\right)
$$

for any $k_{1}>0$. Letting $\tilde{\omega}_{1}=\hat{\omega}_{1}-\omega_{1}$ gives

$$
\dot{x}_{1}=f_{1}\left(\bar{\zeta}_{1}, \chi_{1}, d\right)+\alpha_{1}\left(x_{1}\right)-\rho_{1}\left(y_{1}, \xi_{1}\right) \tilde{\omega}_{1}(\mu) .
$$

Now, it is ready to give the following theorem whose proof can be seen as the special case of that for Theorem 3.2.

Theorem 3.1: Under Assumptions 1.1,1.2, 2.1 and 3.1 for $i=1$, the global regulation problem of the system (1.1) with $r=1$ is solved by the controller (3.7) .

\section{B. The Case with $r \geq 2$}

For the general case with $r>1$, we should go through the recursive operations, during which we will encounter more difficulty caused by the uncertainties propagated from the previous steps. After the recursion, a closed-loop is obtained whose stability can be established by using the certainty equivalence principle. With the functions $\alpha_{i}, i=1, \ldots, r$, and the Lyapunov function $W(\cdot)$ obtained in Section II for the special system satisfying condition (1.3), we can define some notations recursively below which will be utilized later. Also, we can regard the following recursive steps as the controller parameter design algorithm.

\section{Algorithm 3.1: The notations}

$$
\left(\bar{\chi}_{i}\right)-\left(\varrho_{i}, \varpi_{i}\right)-\left(\bar{\zeta}_{i}, \xi_{i}\right)-\left(\rho_{i}, \omega_{i}\right)-\left(\psi_{i}\right)-\left(\lambda_{i}\right)
$$

have been defined in Section III.A for $i=1$ with

$$
\bar{\chi}_{1}=\operatorname{col}\left(x_{o}, \bar{x}_{1}\right), \bar{x}_{1}=x_{1}, \bar{\zeta}_{1}=\bar{z}_{1}, \xi_{1}=s_{1}, \lambda_{1}=\hat{\omega}_{1} .
$$

For convenience, we let $\xi_{o}, \lambda_{o} \in \mathbb{R}^{o}$.

For $i=2, \cdots, r$, the notations (3.8) are defined recursively in the following order:

- $\left(\bar{\chi}_{i}\right): \bar{\chi}_{i}:=\operatorname{col}\left(x_{o}, \bar{x}_{1}, \cdots, \bar{x}_{i}\right)$ where

$$
\bar{x}_{i}=x_{i}+\rho_{i-1}\left(y_{i-1}, \xi_{i-1}, \lambda_{i-2}\right) \hat{\omega}_{i-1}-\alpha_{i-1}\left(\bar{x}_{i-1}\right) .
$$

- $\left(\varrho_{i}, \varpi_{i}\right):$ under Assumption 3.2 and that $\bar{y}_{i}$ contains $\chi_{i}$ as its component, we can define a polynomial function

$$
\begin{array}{r}
\hbar_{i}\left(\bar{y}_{i}, \xi_{i-1}, \lambda_{i-1}, \mu\right)=h_{i}\left(\bar{y}_{i}, \mu\right)+\gamma_{i}\left(\zeta_{i-1}, \chi_{i}, d\right) \\
-\gamma_{i}\left(\bar{\zeta}_{i-1}, x_{o}, \bar{x}_{1}, \bar{x}_{2}+\alpha_{1}\left(\bar{x}_{1}\right), \bar{x}_{i}+\alpha_{i-1}\left(\bar{x}_{i-1}\right), d\right) .
\end{array}
$$


Clearly, the function $\hbar_{i}\left(\bar{y}_{i}, \xi_{i-1}, \lambda_{i-1}, \mu\right)$ is linearly parameterized in the sense that

$$
\hbar_{i}\left(\bar{y}_{i}, \xi_{i-1}, \lambda_{i-1}, \mu\right)=\varrho_{i}\left(\bar{y}_{i}, \xi_{i-1}, \lambda_{i-1}\right) \varpi_{i}(\mu)
$$

for a sufficiently smooth function matrix $\varrho_{i}$ and $a$ column function vector $\varpi_{i}$.

- $\left(\bar{\zeta}_{i}, \xi_{i}\right): \bar{\zeta}_{i}:=\operatorname{col}\left(\bar{z}_{1}, \cdots, \bar{z}_{i}\right)$ and $\xi_{i}:=\operatorname{col}\left(s_{1}, \cdots, s_{i}\right)$ where $s_{i}$ is a square matrix governed by

$$
\dot{s}_{i}=M_{i} s_{i}+\varrho_{i}\left(\bar{y}_{i}, \xi_{i-1}, \lambda_{i-1}\right)
$$

and $\bar{z}_{i}=z_{i}-s_{i} \varpi_{i}(\mu)$.

- $\left(\rho_{i}, \omega_{i}\right):$ By Assumption 1.2, we can denote the time derivative of $\rho_{i-1}\left(y_{i-1}, \xi_{i-1}, \lambda_{i-2}\right)$ by $\bar{\rho}_{i-1}\left(y_{i}, \xi_{i-1}, \lambda_{i-2}, \mu\right)$ since

$$
\begin{array}{r}
\frac{d \rho_{i-1}\left(y_{i-1}, \xi_{i-1}, \lambda_{i-2}\right)}{d t}=\frac{\partial \rho_{i-1}}{\partial y_{i-1}} \varkappa_{i-1}\left(y_{i}, \mu\right) \\
+\sum_{j=1}^{i-1} \frac{\partial \rho_{i-1}}{\partial s_{j}}\left[M_{j} s_{j}+\varrho_{j}\left(\bar{y}_{j}, \xi_{j-1}, \lambda_{j-1}\right)\right] \\
+\sum_{j=1}^{i-2} \frac{\partial \rho_{i-1}}{\partial \hat{\omega}_{j}} \psi_{j}\left(y_{j}, \xi_{j}, \lambda_{j-1}\right) .
\end{array}
$$

Under Assumption 3.1 and that $y_{i}$ contains $\chi_{i}$ as its component, we can define a polynomial function

$$
\begin{array}{r}
\ell_{i}\left(y_{i}, \xi_{i}, \lambda_{i-1}, \mu\right)=l_{i}\left(y_{i}, \mu\right)+f_{i}\left(\zeta_{i}, \chi_{i}, d\right) \\
-f_{i}\left(\bar{\zeta}_{i}, x_{o}, \bar{x}_{1}, \cdots, \bar{x}_{i}+\alpha_{i-1}\left(\bar{x}_{i-1}\right), d\right) \\
+\left(\partial \alpha_{i-1}\left(\bar{x}_{i-1}\right) / \partial \bar{x}_{i-1}\right) \rho_{i-1}\left(y_{i-1}, \xi_{i-1}, \lambda_{i-2}\right) \times \\
\tilde{\omega}_{i-1}(\mu)+\bar{\rho}_{i-1}\left(y_{i}, \xi_{i-1}, \lambda_{i-2}, \mu\right) \hat{\omega}_{i-1} \\
+\rho_{i-1}\left(y_{i-1}, \xi_{i-1}, \lambda_{i-2}\right) \psi_{i-1}\left(y_{i-1}, \xi_{i-1}, \lambda_{i-2}\right), \\
\tilde{\omega}_{i-1}:=\hat{\omega}_{i-1}-\omega_{i-1} .
\end{array}
$$

Clearly, the function $\ell_{i}\left(y_{i}, \xi_{i}, \lambda_{i-1}, \mu\right)$ is linearly parameterized in the sense that

$$
\ell_{i}\left(y_{i}, \xi_{i}, \lambda_{i-1}, \mu\right)=\rho_{i}\left(y_{i}, \xi_{i}, \lambda_{i-1}\right) \omega_{i}(\mu)
$$

for a sufficiently smooth row vector function $\rho_{i}$ and a column function vector $\omega_{i}$.

- $\left(\psi_{i}\right)$ : for any $k_{i}>0$, let

$$
\psi_{i}\left(y_{i}, \xi_{i}, \lambda_{i-1}\right)=k_{i}\left(d W_{i}\left(\bar{x}_{i}\right) / d \bar{x}_{i}\right) \rho_{i}^{\top}\left(y_{i}, \xi_{i}, \lambda_{i-1}\right) .
$$

- $\left(\lambda_{i}\right): \lambda_{i}:=\operatorname{col}\left(\hat{\omega}_{1}, \cdots, \hat{\omega}_{i}\right)$, where $\hat{\omega}_{i}$ is vector variable governed by $\dot{\hat{\omega}}_{i}=\psi_{i}\left(y_{i}, \xi_{i}, \lambda_{i-1}\right)$.

With the above algorithm, it is ready to define the input $u$ from

$$
\bar{x}_{r+1}=u+\rho_{r}\left(y_{r}, \xi_{r}, \lambda_{r-1}\right) \hat{\omega}_{r}-\alpha_{r}\left(\bar{x}_{r}\right)=0 .
$$

To analyze the stability of the closed-loop system, we will first prove the following recursive claim holds. For convenience, the original system (1.1) is decomposed into two parts for a given $(1 \leq j \leq r)$, as denoted by $(1.1)=\Sigma_{j}+$ $\Sigma_{j}^{+}$, where $\Sigma_{j}$ is the subsystem governing $\operatorname{col}\left(\chi_{j}, \zeta_{j}\right)$ and $\Sigma_{j}^{+}$ is that governing $\operatorname{col}\left(\chi_{j}^{+}, \zeta_{j}^{+}\right)$with $\chi_{j}^{+}=\operatorname{col}\left(\chi_{j+1}, \cdots, \chi_{r}\right)$ and $\zeta_{j}^{+}=\operatorname{col}\left(\zeta_{j+1}, \cdots, \zeta_{r}\right)$.
Lemma 3.1: ( $j$-th recursive step $(1 \leq j \leq r)$ ): Suppose the Assumptions 1.1, 1.2, 2.1, 3.1 and 3.2 are satisfied for $i=1, \cdots, j$. Under the following dynamics coordinate transformation

$$
\left(\chi_{r}, \zeta_{r}, \lambda_{j}, \xi_{j}\right) \mapsto\left(\bar{\chi}_{j}, \chi_{j}^{+}, \bar{\zeta}_{j}, \zeta_{j}^{+}, \lambda_{j}, \xi_{j}\right)
$$

as defined in Algorithm 3.1, the original system $\Sigma_{j}+\Sigma_{j}^{+}$ becomes $\bar{\Sigma}_{j}+\Sigma_{j}^{+}$, where $\bar{\Sigma}_{j}$ governing $\operatorname{col}\left(\bar{\chi}_{j}, \bar{\zeta}_{j}\right)$ is given as follows:

$$
\begin{aligned}
\dot{x}_{o}= & f_{o}\left(x_{o}, x_{1}, d\right) \\
\dot{\bar{z}}_{i}= & M_{i} \bar{z}_{i}+\varphi_{i}\left(\bar{\zeta}_{i-1}, \bar{\chi}_{i}, d\right) \\
\dot{\bar{x}}_{i}= & \phi_{i}\left(\bar{\zeta}_{i}, \bar{\chi}_{i}, d\right)+\alpha_{i}\left(\bar{x}_{i}\right)-\rho_{i}\left(y_{i}, \xi_{i}, \lambda_{i-1}\right) \tilde{\omega}_{i}+\bar{x}_{i+1} \\
& i=1, \cdots, j .
\end{aligned}
$$

Proof: We note that the lemma for the first recursive step with $j=1$ has been proved in Section III.A. Now, we suppose the lemma for the $j$-th step holds and will prove it for the $(j+1)$-th step.

First, we note the subsystem for $z_{j+1}$ can be rewritten as follows,

$$
\begin{aligned}
\dot{z}_{j+1}= & M_{j+1} z_{j+1}+\gamma_{j+1}\left(\bar{\zeta}_{j}, x_{o}, \bar{x}_{1}, \bar{x}_{2}+\alpha_{1}\left(\bar{x}_{1}\right), \bar{x}_{j+1}\right. \\
& \left.+\alpha_{j}\left(\bar{x}_{j}\right), d\right)+\hbar_{j+1}\left(\bar{y}_{j+1}, \xi_{j}, \lambda_{j}, \mu\right) \\
= & M_{j+1} z_{j+1}+\varphi_{j+1}\left(\bar{\zeta}_{j}, \bar{\chi}_{j+1}, d\right) \\
& +\hbar_{j+1}\left(\bar{y}_{j+1}, \xi_{j}, \lambda_{j}, \mu\right) .
\end{aligned}
$$

Suppose Assumption 3.2 holds for $i=j+1$. Define the following dynamic coordinate transformation

$$
\begin{aligned}
\bar{z}_{j+1} & =z_{j+1}-s_{j+1} \varpi_{j+1}(\mu) \\
\dot{s}_{j+1} & =M_{j+1} s_{j+1}+\varrho_{j+1}\left(\bar{y}_{j+1}, \xi_{j}, \lambda_{j}\right),
\end{aligned}
$$

under which, we have

$$
\dot{\bar{z}}_{j+1}=M_{j+1} \bar{z}_{j+1}+\varphi_{j+1}\left(\bar{\zeta}_{j}, \bar{\chi}_{j+1}, d\right) .
$$

Furthermore, we have

$$
\begin{array}{r}
\dot{\bar{x}}_{j+1}=f_{j+1}\left(\bar{\zeta}_{j+1}, x_{o}, \bar{x}_{1}, \bar{x}_{2}+\alpha_{1}\left(\bar{x}_{1}\right), \cdots, \bar{x}_{j+1}\right. \\
\left.+\alpha_{j}\left(\bar{x}_{j}\right), d\right)-\left(\partial \alpha_{j}\left(\bar{x}_{j}\right) / \partial \bar{x}_{j}\right)\left(\phi_{j}\left(\bar{\zeta}_{j}, \bar{\chi}_{j}, d\right)\right. \\
\left.+\alpha_{j}\left(\bar{x}_{j}\right)+\bar{x}_{j+1}\right)+\ell_{j+1}\left(y_{j+1}, \xi_{j+1}, \lambda_{j}, \mu\right)+x_{j+2} \\
=\phi_{j+1}\left(\bar{\zeta}_{j+1}, \bar{\chi}_{j+1}, d\right)+\ell_{j+1}\left(y_{j+1}, \xi_{j+1}, \lambda_{j}, \mu\right) \\
+x_{j+2}
\end{array}
$$

where the function $\phi_{j+1}$ is defined in (2.4). If Assumption 3.1 holds for $i=j+1$, we have

$$
\begin{array}{r}
\dot{\bar{x}}_{j+1}=\phi_{j+1}\left(\bar{\zeta}_{j+1}, \bar{\chi}_{j+1}, d\right)+\rho_{j+1}\left(y_{j+1}, \xi_{j+1}, \lambda_{j}\right) \omega_{j+1}(\mu) \\
+x_{j+2} .
\end{array}
$$

Then, let

$$
\begin{aligned}
\bar{x}_{j+2} & =x_{j+2}+\rho_{j+1}\left(y_{j+1}, \xi_{j+1}, \lambda_{j}\right) \hat{\omega}_{j+1}-\alpha_{j+1}\left(\bar{x}_{j+1}\right) \\
\tilde{\omega}_{j+1} & =\hat{\omega}_{j+1}-\omega_{j+1}
\end{aligned}
$$


we have

$$
\begin{aligned}
\dot{\bar{x}}_{j+1}= & \phi_{j+1}\left(\bar{\zeta}_{j+1}, \bar{\chi}_{j+1}, d\right)+\alpha_{J+1}\left(\tilde{x}_{J+1}\right) \\
& -\rho_{j+1}\left(y_{j+1}, \xi_{j+1}, \lambda_{j}\right) \tilde{\omega}_{j+1}+\bar{x}_{j+2} .
\end{aligned}
$$

By noting

$$
\bar{\Sigma}_{j+1}=\bar{\Sigma}_{j}+(3.15)+(3.17),
$$

the lemma for the $(j+1)$-th step has been proved. By mathematical induction, the proof is complete.

Remark 3.1: From the dynamics (3.13) and (3.16) (in particular, the functions $\hbar_{j+1}$ and $\ell_{j+1}$ ), we note that some new uncertainties are incurred, which are propagated from the previous step. And these uncertainties will be further propagated to the subsequent steps when the case $r>2$ is studied. This uncertainty propagation phenomenon significantly complicates the problem.

Observe that the closed-loop system (3.12) reduces to (2.3) when $\tilde{\omega}_{i}=0$. The structure of the closed-loop system (3.12) make it possible to apply the certainty equivalence principle to construct the adaptive law and analyze the stability property. The main result is summarized in the following theorem.

Theorem 3.2: Under Assumptions 1.1, 1.2, 2.1, 3.1 and 3.2 for $i=1, \cdots, r$, the global regulation problem of the system (1.1) is solved by the controller in (3.10) where the parameters are defined in Algorithm 3.1.

Proof: Consider the recursive claim $(r)$ and compare the system (3.12) with $j=r$ and the system (2.3). In Theorem 2.1 , we can define a function

$$
U\left(\bar{\zeta}_{r}, \bar{\chi}_{r}, \tilde{\omega}\right)=V\left(\bar{\zeta}_{r}\right)+W\left(\bar{\chi}_{r}\right)+\frac{1}{2} \tilde{\omega}^{\top} K \tilde{\omega}
$$

with $\tilde{\omega}:=\operatorname{col}\left(\tilde{\omega}_{1}, \cdots, \tilde{\omega}_{r}\right)$. The matrix $K=$ $\operatorname{diag}\left(k_{1}, \cdots, k_{r}\right)$ is diagonal and positive definite which is used to modify the update rate. Then, the derivative of $U\left(\bar{\zeta}_{r}, \bar{\chi}_{r}, \tilde{\omega}\right)$ along the system (3.12) with $j=r$ and $\bar{x}_{r+1}=$ 0 is

$$
\begin{array}{r}
\frac{d U\left(\bar{\zeta}_{r}, \bar{\chi}_{r}, \tilde{\omega}\right)}{d t} \leq-k\left(\bar{\zeta}_{r}, \bar{\chi}_{r}\right)-\frac{\partial W\left(\bar{\chi}_{r}\right)}{\partial\left[\bar{x}_{1}, \cdots, \bar{x}_{r}\right]^{\top}} \times \\
\rho\left(y_{r}, \xi_{r}, \lambda_{r-1}\right) \tilde{\omega}+\tilde{\omega}^{\top} K \psi\left(y_{r}, \xi_{r}, \lambda_{r-1}\right)
\end{array}
$$

where

$$
\begin{aligned}
\rho\left(y_{r}, \xi_{r}, \lambda_{r-1}\right) & =\operatorname{diag}\left(\rho_{1}\left(y_{1}, \xi_{1}, \lambda_{o}\right), \cdots, \rho_{r}\left(y_{r}, \xi_{r}, \lambda_{r-1}\right)\right) \\
\psi\left(y_{r}, \xi_{r}, \lambda_{r-1}\right) & =\operatorname{col}\left(\psi_{1}\left(y_{1}, \xi_{1}, \lambda_{o}\right), \cdots, \psi_{r}\left(y_{r}, \xi_{r}, \lambda_{r-1}\right)\right) .
\end{aligned}
$$

Now, let the update law be $\dot{\tilde{\omega}}=\psi\left(y_{r}, \xi_{r}, \lambda_{r-1}\right)$. As a result,

$$
\frac{d U\left(\bar{\zeta}_{r}, \bar{\chi}_{r}, \tilde{\omega}\right)}{d t} \leq-k\left(\bar{\zeta}_{r}, \bar{\chi}_{r}\right) .
$$

Thus, the states $\bar{\zeta}_{r}, \bar{\chi}_{r}$, and $\tilde{\omega}$ are bounded. It is easy to verify $\xi_{r}$ is bounded. In other words, all states in the closed-loop system are bounded. Next, let $U(t)=U\left(\bar{\zeta}_{r}(t), \bar{\chi}_{r}(t), \tilde{\omega}(t)\right)$ and $k(t)=k\left(\bar{\zeta}_{r}(t), \bar{\chi}_{r}(t)\right)$. Integrating on both sides of (3.18) shows that $\int_{o}^{t} k(\tau) d \tau \leq U(0)-U(t) \leq U(0)$, hence, $\lim _{t \rightarrow \infty} \int_{o}^{t} k(\tau) d \tau$ exists and is finite. The fact that all states are bounded implies that

$$
\dot{k}(t)=\frac{\partial k\left(\bar{\zeta}_{r}, \bar{\chi}_{r}\right)}{\partial \bar{\zeta}_{r}} \dot{\bar{\zeta}}_{r}+\frac{\partial k\left(\bar{\zeta}_{r}, \bar{\chi}_{r}\right)}{\partial \bar{\chi}_{r}} \dot{\bar{\chi}}_{r}
$$

is bounded for all $t \geq 0$, hence, $k(t)$ is uniformly continuous. By Barbalat's lemma, $\lim _{t \rightarrow \infty} k(t)=0$, hence $\lim _{t \rightarrow \infty} e(t)=0$. The proof is thus completed.

\section{An EXAMPLE}

As mentioned in the Introduction, the regulation problem studied in this paper is motivated from studying the global robust servomechanism problem of lower triangular nonlinear systems subject to an uncertain linearly neutrally stable exosystem. The e.p.p. terms $g_{i}$ and $p_{i}$ reflect the mismatch between the actual unknown parameter and its estimated value in the exosystem. Due to the space limit, we cannot give a full treatment of this problem. We will instead consider a specific second order lower triangular system as follows:

$$
\begin{aligned}
& \dot{q}_{o}=-q_{o}+0.2 e, \quad \dot{q}_{1}=0.5 q_{o} q_{1}+w_{1} q_{1}+q_{2} \\
& \dot{q}_{2}=w_{2} q_{o}^{2}+w_{3} q_{1}+u, \quad e=q_{1}-v_{1}
\end{aligned}
$$

where $v_{1}$ is generated by an exosystem

$$
\dot{v}_{1}=-\sigma v_{2}, \dot{v}_{2}=\sigma v_{1}
$$

with $\sigma$ an unknown parameter. These equations formulate the control problem of designing a state-feedback regulator to have the state $q_{1}$ asymptotically converges to $v_{1}$, i.e., $\lim _{t \rightarrow \infty} e(t)=0$. The system contains unknown parameters $\left(w_{1}, w_{2}, w_{3}\right)$ and $\sigma$. We will first show that this problem can be converted into the global regulation problem of a system in the form of (1.1) following the general framework of [10].

First, let

$$
\begin{aligned}
& \mathbf{q}_{1}(v, w, \sigma)=v_{1} \\
& \mathbf{q}_{2}(v, w, \sigma)=-\sigma v_{2}-w_{1} v_{1} \\
& \mathbf{q}_{2}(v, w, \sigma)=-\sigma^{2} v_{1}+w_{1} \sigma v_{2}-w_{3} v_{1} .
\end{aligned}
$$

Then $\left(\mathbf{q}_{1}, \mathbf{q}_{2}, \mathbf{u}\right)$ where $\mathbf{u}=\mathbf{q}_{2}$ constitutes the solution of the regulator equations of system (4.1). Next we need to design internal models corresponding to the state $q_{2}$ and input $u$. For this purpose, note that $\mathbf{q}_{2}$ and $\mathbf{q}_{3}$ are linear in $v$, both the state $x_{2}$ and the input $u$ admit a linear internal model of dimension two as follows:

$$
\dot{\eta}_{i}=M_{i} \eta_{i}+N_{i} q_{i+1}, i=1,2
$$

where $M_{i}$ is any Hurwitz matrix and $N_{i}$ is any column vector such that $\left(M_{i}, N_{i}\right)$ is controllable. In order to convert the output regulation problem of the given plant into a regulation problem of an augmented system, we need to introduce a set of canonical coordinate transformation. For this purpose, we first define some notations. For $i=1,2$, let

$$
\Phi_{i}(\sigma)=\left[\begin{array}{cc}
0 & 1 \\
-\sigma^{2} & 0
\end{array}\right]
$$

and

$$
M_{i}=\left[\begin{array}{cc}
-1 & 0 \\
0 & -2
\end{array}\right], N_{i}=\left[\begin{array}{l}
0.2 \\
0.5
\end{array}\right]
$$


Solving

$$
T_{i}(\sigma) \Phi_{i}(\sigma)-M_{i} T_{i}(\sigma)=N_{i}\left[\begin{array}{llll}
1 & 0 & \cdots & 0
\end{array}\right]
$$

gives

$$
T_{i}(\sigma)=\left[\begin{array}{ll}
\frac{0.2}{\sigma^{2}+1} & -\frac{0.2}{\sigma^{2}+1} \\
\frac{1}{\sigma^{2}+4} & -\frac{0.5}{\sigma^{2}+4}
\end{array}\right] .
$$

Finally, define

$$
\begin{aligned}
\theta_{i}(v, w, \sigma) & =T_{i}(\sigma) \operatorname{col}\left(\mathbf{q}_{i+1}, \dot{\mathbf{q}}_{i+1}\right) \\
E_{i}(\sigma) & :=T_{i}(\sigma) \Phi_{i}(\sigma) T_{i}^{-1}(\sigma) \\
\Psi_{i}(\sigma) & :=\left[\begin{array}{llll}
1 & 0 & \cdots & 0
\end{array}\right] T_{i}^{-1}(\sigma) .
\end{aligned}
$$

Now applying the following coordinate transformation

$$
x_{o}=q_{o}, x_{1}=e, x_{2}=q_{2}, z_{i}=\eta_{i}-\theta_{i}-N_{i} x_{i}, i=1,2
$$

to the system composed of the given plant (4.1) and the internal models (4.3) yields a system of the form (1.1) with

$$
\begin{aligned}
f_{o} & =-x_{o}+0.2 x_{1}, \quad \gamma_{1}=M_{1} N_{1} x_{1}-N_{1} A_{1} \\
g_{1} & =0, f_{1}=A_{1}+\Psi_{1}(\sigma) \eta_{1}-\Psi_{1}(\sigma) \theta_{1} \\
p_{1} & =-\Psi_{1}(\sigma) \eta_{1} \\
\gamma_{2} & =M_{2} N_{2} x_{2}-N_{2} A_{2}+N_{2} \Psi_{1}(\sigma) E_{1}(\sigma)\left(N_{1} x_{1}+z_{1}\right) \\
g_{2} & =-N_{2} \Psi_{1}(\sigma) E_{1}(\sigma) \eta_{1} \\
f_{2} & =A_{2}-\Psi_{1}(\sigma) E_{1}(\sigma)\left(N_{1} x_{1}+z_{1}\right)+\Psi_{2}(\sigma)\left(N_{2} x_{2}+z_{2}\right) \\
p_{2} & =\Psi_{1}(\sigma) E_{1}(\sigma) \eta_{1}-\Psi_{2}(\sigma) \eta_{2} \\
d & =\operatorname{col}(w, w, \sigma), \mu=\operatorname{col}(w, \sigma)
\end{aligned}
$$

and $A_{1}:=0.5 x_{o}\left(x_{1}+v_{1}\right)+w_{1} x_{1}, \quad A_{2}:=w_{2} x_{o}^{2}+$ $w_{3} x_{1}$. The measurement outputs can be defines as follows: $\bar{y}_{1} \in \mathbb{R}^{o}, y_{1}=\eta_{1}, \bar{y}_{2}=\operatorname{col}\left(e, q_{o}, q_{1}, q_{2}, \eta_{1}\right)$, and $y_{2}=$ $\operatorname{col}\left(e, q_{o}, q_{1}, q_{2}, \eta_{1}, \eta_{2}\right)$. It can be seen that the solvability of the global regulation problem for the system (1.1) using the measurement outputs will imply the solvability of the servomechanism problem of the system (4.1). It is possible to verify that all assumptions in Theorem 3.2 are satisfied and thus the global regulation problem for the system is solvable. To explicitly give the controller (3.10), we can make use of Algorithm 3.1 to find all parameters. The details are omitted due to the space limit.

In the numerical simulation, we can compare the nonadaptive controller and the adaptive one. The simulation is conducted with the parameters $w_{1}=-0.4, w_{2}=0.8, w_{3}=$ $0.3, v_{1}(0)=10, v_{2}(0)=0, q_{o}(0)=5, q_{1}(0)=8, q_{2}(0)=$ -1 , and the initial values of the remaining states being zero. The tracking performance is shown in Figure. 1. For the first 100 seconds, the value of $\sigma=0.2$ is the same as that used for the controller design, the adaptive law is off. The desired tracking performance $\lim _{t \rightarrow \infty} e(t)=0$ is achieved. At $t=100$, the parameter $\sigma$ changes its value to $\sigma=1$ and the tracking performance degrades significantly. When the adaptive law is turned on at $t=200$, the tracking error quickly converges to zero. Good tracking performance is maintained even after another step change of the parameter to $\sigma=0.2$ at $t=300$.
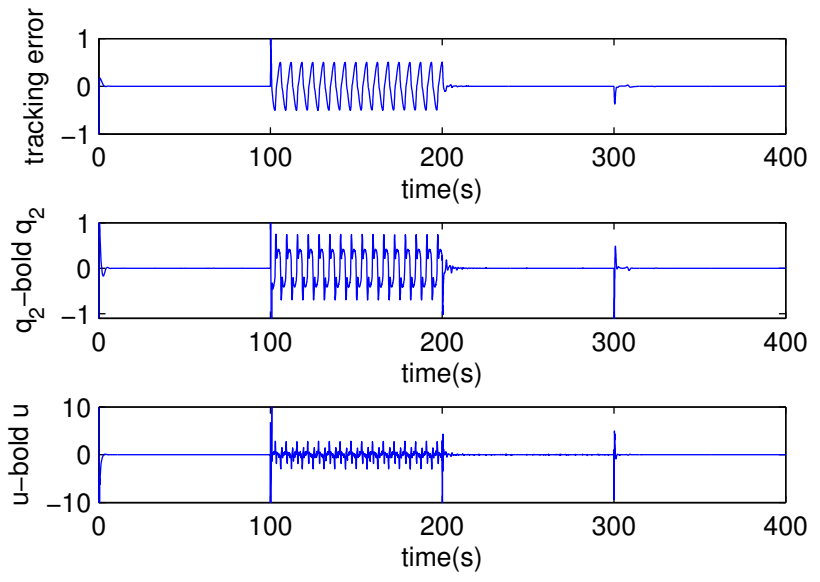

Fig. 1. Profile of the tracking errors

\section{CONClusion}

In this paper, we have presented a set of sufficient conditions for the solvability of a global regulation problem for cascaded polynomial systems containing dynamic uncertainties. A framework combining both robust and adaptive techniques has been developed. The global regulation problem is motivated from the global servomechanism problem for the lower triangular systems subject to an uncertain exosystem. A simple example has been given to illustrate this point. It is interesting to further investigate the application of this paper to solving the general global servomechanism problem for the lower triangular systems subject to an uncertain exosystem.

\section{REFERENCES}

[1] Z. P. Jiang and I. Mareels. A small-gain control method for nonlinear cascaded systems with dynamic uncertainties. IEEE Transactions on Automatic Control, 42:292-308, 1997.

[2] Z. P. Jiang and L. Praly. Design of robust adaptive controllers for nonlinear systems with dynamic uncertainties. Automatica, 34:825840, 1998.

[3] Z. Chen and J. Huang. Dissipativity, stabilization, and regulation of cascade-connected systems. IEEE Transactions on Automatic Control, 49:635-650, 2004.

[4] Z. Chen and J. Huang. A Lyapunov's direct method for the global robust stabilization of nonlinear cascaded systems. Automatica, 44:745-752, 2008.

[5] H. Khalil. On the design of robust servomechanisms for minimum phase nonlinear systems. Int. J. Robust Nonlinear Control, 10:339$361,2000$.

[6] C. I. Byrnes and A. Isidori. Limit sets, zero dynamics and internal models in the problem of nonlinear output regulation. IEEE Transactions on Automatic Control, 48:1712-1723, 2003.

[7] Z. Ding. Global stabilization and disturbance suppression of a class of nonlinear systems with uncertain internal model. Automatica, 39:471479, 2003.

[8] A. Pavlov, N. van de Wouw, and H. Nijmeijer. Uniform Output Regulation of Nonlinear Systems: a Convergent Dynamics Approach. Birkhauser, 2006.

[9] Z. Chen. Global stabilization of nonlinear cascaded systems with a lyapunov function in superposition form. Automatica, 2009. DOI: 10.1016/j.automatica.2009.04.021.

[10] J. Huang and Z. Chen. A general framework for tackling the output regulation problem. IEEE Transactions on Automatic Control, 49:2203-2218, 2004. 